\title{
Current status of development of anticancer agents in Japan
}

\author{
Tomohiro Morita · Akiko Hori · Hiroto Narimatsu • \\ Tetsuya Tanimoto $\cdot$ Masahiro Kami
}

Published online: 22 May 2008

(C) The Japanese Society of Hematology 2008

\section{Erratum to: Int J Hematol}

\section{DOI 10.1007/s12185-008-0087-2}

The correct name of the third author should be given as Hiroto Narimatsu, not Hiroto Narimatatsu.

The online version of the original article can be found under doi:10.1007/s12185-008-0087-2.

T. Morita $(\bowtie) \cdot$ H. Narimatsu $\cdot$ M. Kami

Division of Exploratory Research,

The Institute of Medical Science,

The University of Tokyo, 4-6-1, Shirokanedai,

Minato-ku, Tokyo 108-8639, Japan

e-mail: t.morita526@gmail.com

A. Hori

First Outpatient Division,

The National Cancer Center Hospital,

Tokyo, Japan

T. Tanimoto

Department of Laboratory Medicine,

The National Cancer Center Hospital, Tokyo, Japan 